\title{
Risk and protective factors for structural brain ageing in the eighth decade of life
}

\author{
Stuart J. Ritchie ${ }^{1,2} \cdot$ Elliot M. Tucker-Drob $^{3} \cdot$ Simon R. Cox $^{1,2} \cdot$ David Alexander Dickie, Di,6, $_{\text {. }}$ \\ Maria del C. Valdés Hernández ${ }^{2,4,5,6} \cdot$ Janie Corley $^{1,2} \cdot$ Natalie A. Royle $^{2,4,5,6} \cdot$ Paul Redmond $^{1}$ • \\ Susana Muñoz Maniega ${ }^{2,4,5,6} \cdot$ Alison Pattie $^{1} \cdot$ Benjamin S. Aribisala $^{2,4,5,6,7} \cdot$ Adele M. Taylor $^{1} \cdot$ Toni-Kim Clarke $^{8}$. \\ Alan J. Gow ${ }^{2,9} \cdot$ John M. Starr ${ }^{2,10} \cdot$ Mark E. Bastin ${ }^{2,4,5,6} \cdot$ Joanna M. Wardlaw ${ }^{2,4,5,6} \cdot$ Ian J. Deary ${ }^{1,2}$
}

Received: 1 December 2016 / Accepted: 27 March 2017 / Published online: 19 April 2017

(C) The Author(s) 2017. This article is an open access publication

\begin{abstract}
Individuals differ markedly in brain structure, and in how this structure degenerates during ageing. In a large sample of human participants (baseline $n=731$ at age 73 years; follow-up $n=488$ at age 76 years), we estimated the magnitude of mean change and variability in changes in MRI measures of brain macrostructure (grey matter, white matter, and white matter hyperintensity volumes) and microstructure (fractional anisotropy and mean diffusivity from diffusion tensor MRI). All indices showed significant average change with age, with considerable heterogeneity in those changes. We then tested eleven socioeconomic, physical, health, cognitive, allostatic (inflammatory and metabolic), and genetic variables for their value in predicting these differences in changes. Many of these variables were significantly correlated with baseline brain structure, but few could account for significant portions of the heterogeneity in subsequent brain change. Physical fitness was an exception, being correlated both with brain level and
\end{abstract}

Electronic supplementary material The online version of this article (doi:10.1007/s00429-017-1414-2) contains supplementary material, which is available to authorized users.

Stuart J. Ritchie

stuart.ritchie@ed.ac.uk

1 Department of Psychology, The University of Edinburgh, Edinburgh, UK

2 Centre for Cognitive Ageing and Cognitive Epidemiology, The University of Edinburgh, Edinburgh, UK

3 Department of Psychology, University of Texas, Austin, TX, USA

4 Brain Research Imaging Centre, The University of Edinburgh, Edinburgh, UK

5 Scottish Imaging Network, A Platform for Scientific Excellence (SINAPSE) Collaboration, Edinburgh, UK changes. The results suggest that only a subset of correlates of brain structure are also predictive of differences in brain ageing.

Keywords Ageing · Longitudinal · Structural MRI · Genetic $\cdot$ Lifestyle $\cdot$ Prediction

\section{Introduction}

Human brain structure shows several changes as people age. Healthy brain tissue atrophies in later life, with average losses of around $0.5 \%$ of total brain volume per year (Fotenos et al. 2005). Features such as white matter hyperintensities increase in size (Schmidt et al. 2005). Changes in white matter microstructure, measured using diffusion tensor (DT) MRI, also occur, with white matter tracts showing decreases in fractional anisotropy (FA; indicating the directional coherence of the movement of water molecules in the tracts; Kochunov et al. 2012) and increases in mean diffusivity (MD; indicating the average molecular motion in

6 Centre for Clinical Brain Sciences, The University of Edinburgh, Edinburgh, UK

7 Computer Science Department, Faculty of Science, Lagos State University, Lagos, Nigeria

8 Division of Psychiatry, The University of Edinburgh, Edinburgh, UK

9 Department of Psychology, Heriot-Watt University, Edinburgh, UK

10 Alzheimer Scotland Dementia Research Centre, The University of Edinburgh, Edinburgh, UK 
the tracts; Hsu et al. 2010; Muñoz Maniega et al. In press). Alterations in these key neural parameters have been linked to decreases in cognitive ability (Kloppenborg et al. 2014; Lövdén et al. 2014; Ritchie et al. 2015a, b), and thus may form part of the neural basis of ageing-related cognitive decline (Salthouse 2011). Since cognitive decline-even without dementia-is an increasing economic and social burden in Western countries (Brayne 2007), understanding the predictors of later-life brain changes is of growing importance. Understanding why some individuals' brains age more healthily than others' - that is, investigating what some researchers have termed "brain maintenance" (Nyberg et al. 2012) - is a critical step toward designing treatments and interventions to ameliorate cognitive decline.

Previous cross-sectional studies have discovered correlations between differences in adult brain macro- and microstructure and lifestyle factors such as smoking and alcohol consumption (Hudkins et al. 2012; Oscar-Berman and Marinković 2007), health conditions (Qiu 2014; Raz et al. 2003), physical fitness (Sexton et al. 2015), allostatic load (a measure of accumulated stress; Booth et al. 2015), and genetic factors such as the $A P O E$ e4 allele (Laukka et al. 2015) and genetic risk for schizophrenia (Staal et al. 2000). Although there is mixed evidence regarding this latter association (Liu et al. 2016; van der Auwera et al. 2015), there is reason to hypothesize that a higher load of schizophrenia-related genes may predispose individuals to poorer brain health, since strong genetic correlations have been found between schizophrenia and various cognitive abilities (Hagenaars et al. 2016).

Importantly, only some of these variables have been investigated as predictors of longitudinal changes in brain structure, and they have rarely been examined simultaneously. Poorer physical fitness (Tian et al. 2015), higher levels of glycated haemoglobin (Enzinger et al. 2005), and hypertension (Raz et al. 2003) have individually been associated with accelerated rates of brain atrophy. There is conflicting evidence for the role of the APOE e4 allele in brain tissue loss in individuals without dementia (Josephs et al. 2008; Wishart et al. 2006). Risk factors for the accumulation of white matter hyperintensities, which have been studied in more depth than changes in healthy brain tissues, tend to be vascular-related (Wardlaw et al. 2015). Very few longitudinal studies have examined predictors of later-life change in white matter diffusion parameters (see Köhncke et al. 2016, for the one example of which we are aware). The distinction between cross-sectional and longitudinal analyses of brain ageing is important: in cross-sectional studies, it is not possible to differentiate between developmental processes that occur in earlier periods of life from effects that are specifically ageing-related (Tucker-Drob and Salthouse 2011).
The present study reports an analysis of predictors of change in brain volumes (grey matter, normal-appearing white matter, and white matter hyperintensities) and diffusion parameters (latent general factors of fractional anisotropy and of mean diffusivity, extracted from diffusion tensor imaging of multiple white matter tracts across the brain; see Methods) between age 73 and 76 years. We selected a wide range of potential predictors on the basis of the literature surveyed above, examining health, fitness, lifestyle, cognitive, socioeconomic, and genetic variables. In order to reduce the numerous predictor variables to a manageable number, where possible we extracted latent variables that indexed shared variance between groups of predictors. The final predictor list was the following: sex, physical fitness, allostatic load, health conditions, socioeconomic status, prior intelligence, education, smoking, alcohol consumption, $A P O E$ e 4 status, and polygenic risk for schizophrenia. We tested the predictors' relation to brain changes. First, we examined the value of each predictor individually. Second, we used penalized regression (Zou and Hastie 2005) to create parsimonious multivariate models that predicted each brain outcome from an optimal combination of predictors.

\section{Materials and methods}

\section{Participants}

The Lothian Birth Cohort 1936 (Deary et al. 2007, 2012) is a longitudinal study of ageing based in the Edinburgh and Lothians area of Scotland, United Kingdom. Participants were invited to join the study on the basis of their participation, at age 11, in the Scottish Mental Survey of 1947, during which most had completed a test of general intelligence (see below). They were followed up in later life and have so far completed three waves of later-life testing. At the first wave, in 2004-2007, participants were aged 69.53 years on average ( $\mathrm{SD}=0.83 ; n=1,091 ; 543$ females). At the second wave, in 2007-2010, they were aged 72.49 years ( $\mathrm{SD}=0.71 ; n=866 ; 418$ females). At the third wave, in 2011-2014, they were aged 76.25 years $(S D=0.68$, $n=697 ; 337$ females). The majority of the 'predictor' variables in the present study, described below, were measured at the second testing wave.

At the second and third waves, the participants were also invited to attend for a structural and diffusion tensor brain scan (Wardlaw et al. 2011). 731 participants (343 females) were scanned at the second wave (mean age: 72.68 years; $\mathrm{SD}=0.72$ ), and 488 (228 females) were scanned at the third wave (mean age: 76.38 years; $\mathrm{SD}=0.65$ ). Different amounts of usable data were obtained for each of the brain measures: the valid sample sizes for each measure are shown in Table 2. 
The study was approved by the Multi-Centre Research Ethics Committee for Scotland (MREC/01/0/56) and the Lothian Research Ethics Committee (LREC/2003/2/29). All participants completed written informed consent forms before any cognitive, MRI, or other measurements were taken.

\section{Measures}

\section{MRI scanning}

All participants were scanned using the same scanner (1.5 T GE Signa Horizon HDx; General Electric, Milwaukee, WI, USA), using the same protocol, at both waves. The full imaging protocol is described elsewhere (Wardlaw et al. 2011).

\section{Brain volumetric measures}

We measured brain volumes (specifically grey matter, normal-appearing white matter, and white matter hyperintensity volumes) using a validated multispectral image processing method that combines T1-, T2-, T2*-, and FLAIRweighted MRI sequences for segmentation (based on a previous method; Valdés Hernández et al. 2010). According to STandards for ReportIng Vascular changes on $\mathrm{nEu}-$ roimaging (STRIVE), we explicitly defined white matter hyperintensities as punctate, focal, or diffuse lesions in all subcortical regions (Wardlaw et al. 2013). We manually checked all segmented images for accuracy blinded to all participant characteristics, corrected errors, and excluded imaging-detected infarcts from white matter hyperintensity volumes (Wang et al. 2012). White matter hyperintensity volume was log-transformed before inclusion in the analyses, since it had a right-skewed distribution (that is, many participants had few hyperintensities). White matter hyperintensities are illustrated as the crosshatched areas in Fig. 1, alongside the diffusion-tensor measures described below. Note that the analyses below are performed on the total tissue volumes; when adjusting these volumes for intracranial volume, the pattern of results-especially for longitudinal changes (the main focus of the report) — was nearly identical.

\section{White matter diffusion-tensor measures}

All diffusion MRI data were converted from DICOM (http://dicom.nema.org/) to NIfTI-1 (http://nifti.nimh. nih.gov/nifti-1/) format using TractoR tools (http://www. tractor-mri.org.uk; Clayden et al. 2011), and pre-processed with FSL tools (http://www.fmrib.ox.ac.uk/fsl/; Jenkinson et al. 2012) in order to extract the brain, eliminate bulk patient motion and eddy current-induced artifacts, and estimate FA and MD in each brain voxel. The underlying connectivity data was generated using BedpostX/ProbTrackX (Behrens et al. 2007), with a two-fiber model and 5000 streamlines to reconstruct tracts of interest.

As described in detail previously (Ritchie et al. 2015a), 12 tracts of interest were identified in each participant using probabilistic neighborhood tractography, an automatic tract segmentation method with good reproducibility (Clayden et al. 2009). The 12 tracts segmented were the genu and splenium of corpus callosum; the bilateral cingulum cingulate gyri; the arcuate, uncinate, and inferior longitudinal fasciculi; and the anterior thalamic radiations. Tract-averaged values of MD and FA, weighted by connection probability, were generated for each pathway in those tracts that were visually assessed to be anatomically plausible representations of the fasciculus of interest. The tracts are illustrated in color in Fig. 1.
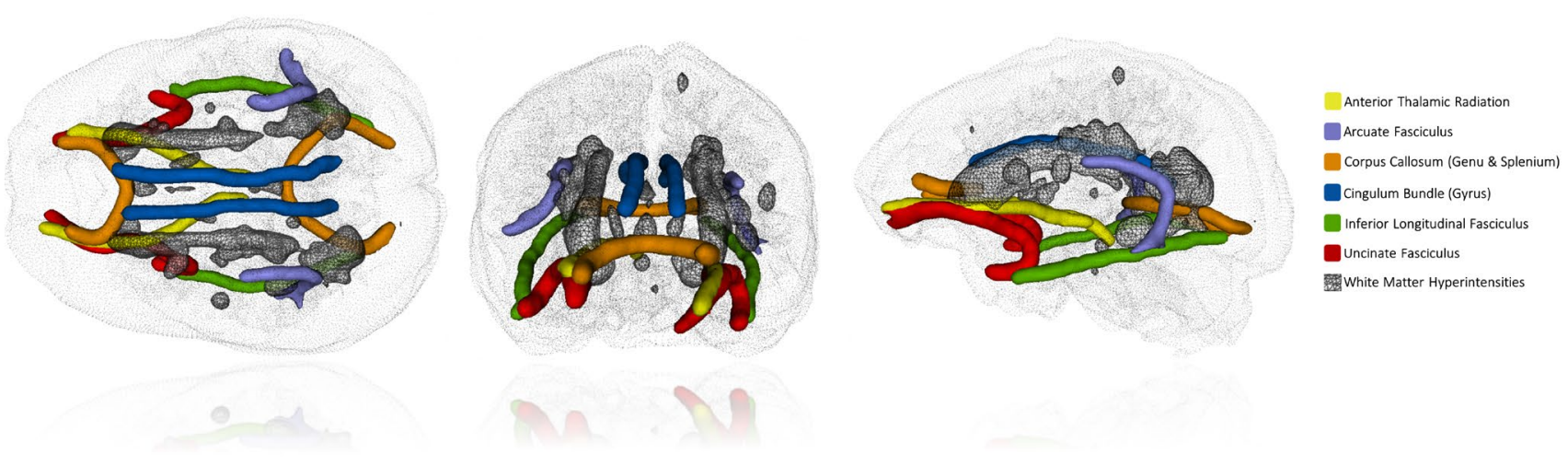

Fig. 1 Illustration of the 12 white matter tracts (five bilateral; two from the corpus callosum) measured using probabilistic neighborhood tractography in one Lothian Birth Cohort 1936 study participant (in color). Also shown are the volumetrically-estimated white matter hyperintensities observed in this participant (crosshatched). Tracts and hyperintensities are displayed inside the participant's spatiallyregistered T1-weighted brain volume 
At each scanning wave, we estimated latent general factors of FA and MD from tract-averaged estimates from each of the measured tracts. These general factors capture the shared variance in FA or MD across multiple tracts. Similar analyses have been used in several previous studies of white matter microstructure (Ritchie et al. 2015a; Bender et al. 2016). All latent variable loadings were highly significant and mostly moderate-to-large in size, except for the loading of MD in the splenium of the corpus callosum on general MD at each wave, which were weak $(0.200$ at age 73 and 0.275 at age 76 ). For this reason, we did not use this tract as part of the general MD factor, reducing the number of indicators for MD at each wave to 11. All 12 tracts were used to indicate the general FA factor at both waves.

\section{Predictors of brain change}

We selected 11 candidate predictors of brain change. For four of the predictors, we created latent variables from multiple manifest indicators. Physical fitness comprised grip strength in the dominant hand (measured on a Hydraulic Hand Dynamometer), forced expiratory volume in 1s (measured using a Micro Medical Spriometer), and 6-metre walk time, all measured at age 73 . All three physical variables were adjusted for height. Allostatic load, a variable theoretically representing 'wear and tear' on bodily systems (McEwen 1998), was indicated by a variety of inflammatory biomarkers (fibrinogen, C-reactive protein, interleukin-6) and metabolic biomarkers (triglycerides, glycated haemoglobin, low- and high-density lipoprotein, and body mass index), all derived from blood samples or direct measurements taken at age 73 (Booth et al. 2015). Socioeconomic status was indicated by the participant's father's occupational class at their birth in 1936, the participant's own highest achieved occupational class before retirement, and the Scottish Index of Multiple Deprivation, a government-collected, neighborhood-level indicator of social deprivation, estimated for the present participants at age 70 (Executive 2006). Prior intelligence was indicated by the participant's scores on three tests: the Moray House Test No. 12 (Scottish Council for Research in Education 1949), taken at age 11, the National Adult Reading Test (Nelson and Willison 1991), and the Wechsler Test of Adult Reading (Wechsler 2001). The latter two tests were taken at age 70 , during the first wave of the study.

The remaining seven predictors were included in the model as manifest (i.e. single) variables. Sex was indicated at entry to the study. Health conditions were measured as a sum of the number of the following conditions the participants reported suffering from: diabetes, hypertension, stroke, and cardiovascular disease (since these were all binary variables, we chose to calculate a sum rather than a latent variable). Education was self-reported by the participants as the number of years of formal, fulltime education the participants completed. Smoking status was recorded as current, ex-, or never, reported at age 73 . Alcohol consumption was recorded as grams per week, as reported on a validated Food Frequency Questionnaire at age 70 (this variable was log-transformed to normalize its distribution). Apolipoprotein (APOE) e4 genotype was derived from blood samples using TaqMan technology at the Wellcome Trust Clinical Research Facility Genetics Core at the Western General Hospital, Edinburgh. The $A P O E$ variable used here classifies participants into 'no e4 alleles' versus 'one or two e4 alleles'. Finally, each participant's regression-weighted polygenic risk score for schizophrenia was derived from single-nucleotide polymorphism (SNP) genotyping, using summary data from the most recent genome-wide association study for schizophrenia (Schizophrenia Working Group of the Psychiatric Genomics Consortium 2014; see previous work in this sample by McIntosh et al. 2013, for more details about risk score calculation). For the present study, we used the most liberal SNP inclusion threshold (all SNPs, $p=1.00$ ). When using the polygenic score, we also included four multidimensional scaling components to control for population stratification. Participants also completed the Mini-Mental State Examination (MMSE; Folstein et al. 1975) at both waves. This was used as part of a secondary analysis, described below.

\section{Statistical analyses}

We estimated three structural equation models, each including different brain measurements as the dependent variables. These were change score models (McArdle 2009), which allow the assessment of levels (in this case, the brain's baseline status at age 73) and changes across two measurement waves (brain changes between age 73 and age 76).

Model 1 had as its dependent variables three brain volumetric changes: changes in the volumes of grey matter, normal-appearing white matter, and white matter hyperintensities. Model 2 used the FA values from the DT-MRI scans, producing a latent variable of general FA across the 12 tracts at each scanning wave, and a latent change score variable $(\Delta \mathrm{FA})$ indexing the FA change across the waves. Model 3 used the MD values from the same DTMRI scans, producing a latent variable of cross-wave MD change $(\Delta \mathrm{MD})$. Figure 2 shows a simplified diagram of the model. For general FA and MD, we imposed strong measurement invariance across the waves (that is, we constrained the loadings and intercepts of each tract to equality across wave, under the assumption that the same latent trait was being measured across waves; see Widaman et al. 2010; Ritchie et al. 2015a) Since there was a small 
Fig. 2 Simplified diagram of the structural equation model for the general fractional anisotropy $(F A)$ variable. FA in white matter tracts $1-12$ was measured at baseline (age 73) and follow-up (age 76), and a General FA factor extracted at each wave. From these a latent change score variable ( $\Delta$ General FA) was calculated. Then, each of the predictor variables was assessed for its relation with baseline level (path $A$ ) and change (path $B$ ). The equivalent model for general mean diffusivity used only 11 tracts. The equivalent models for grey matter volume, white matter volume, and white matter hyperintensity volume did not use latent factors at each wave

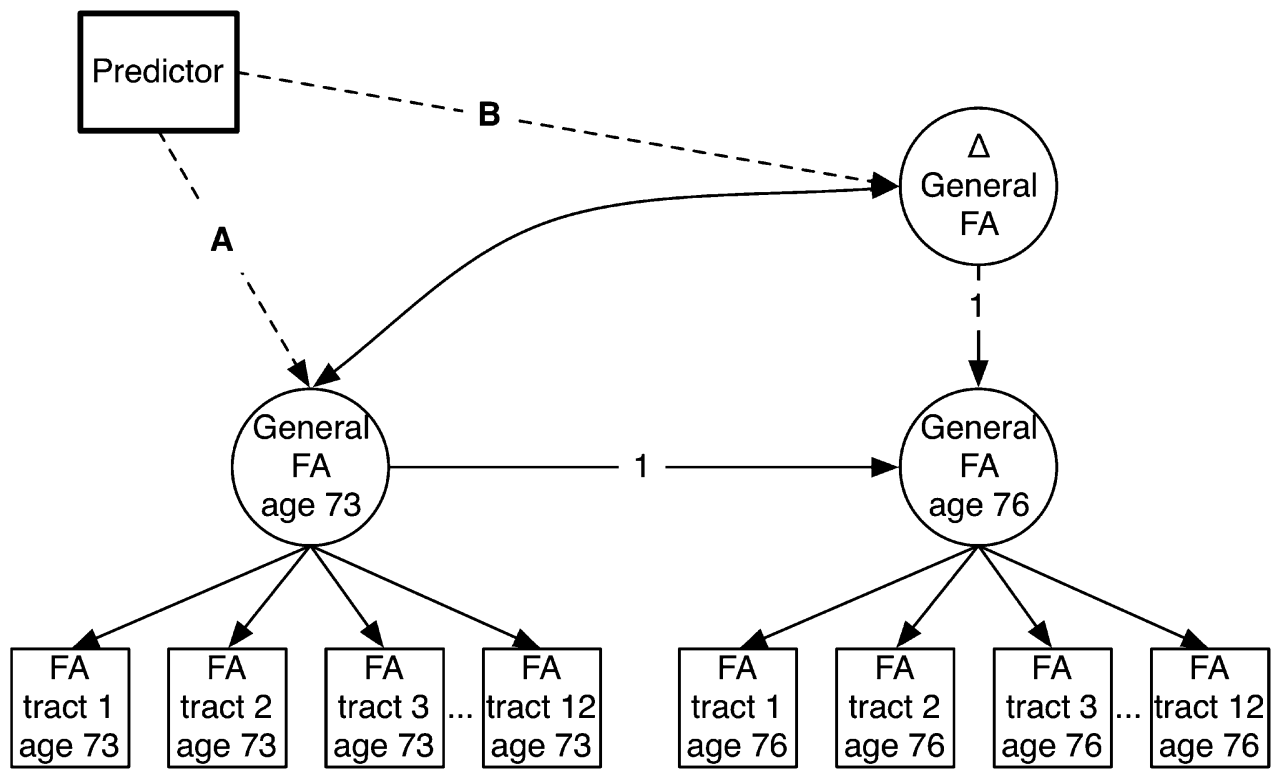

amount of within-wave age variability, models adjusted the brain variables for the participant's age in days at the time of scanning. For each of the three models, we tested the association of brain baseline levels and changes with each individual predictor variable (some of which were latent variables, and some of which were manifest, as described above), by regressing the brain outcome on each predictor separately. All of these analyses also adjusted for sex. Structural equation modeling was performed in Mplus v7.3 (Muthén and Muthén 1998-2014), and used full-information maximum likelihood estimation in order to use all of the available data.

Next, we produced simultaneous, multivariate regression models that included a combination of the most appropriate predictor variables for each brain outcome. Methods of producing such models include forwards and backwards stepwise regression, but these can lead to overfitting, and thus a lower likelihood of findings that are replicable in independent datasets. For this reason, we used a penalized regression method, the elastic net (Zou and Hastie 2005), which combines the advantages of least absolute shrinkage and selection operator (LASSO) regression (production of a parsimonious model by removing irrelevant variables) and ridge regression (good performance in the presence of collinear predictors, as was the case in our dataset; Morozova et al. 2015). These analyses were performed using the 'glmnet' package for R (Friedman et al. 2010), with 10-fold cross-validation. We bootstrapped each model 1000 times, including in the final model only those variables that had a non-zero coefficient in $50 \%$ or more of the iterations. Thus, we selected variables that were stably chosen by the estimator as relevant predictors of each neuroanatomical outcome. We took these selected predictors and simultaneously entered them into the latent difference score models to produce the results described below.

\section{Results}

Full descriptive statistics and valid sample sizes for all measures, along with the loadings of each variable on its factor (where applicable) are presented in Tables 1 (for predictors) and 2 (for brain parameters). Correlations among the predictor variables are shown in Table 3 .

All brain measures showed statistically significant mean change across the 3 years of the study (all $p$ values $<0.001)$. The models implied that grey matter volume change was $-0.07 \mathrm{SDs} / y e a r(-0.64 \%$ change/year). Normal-appearing white matter volume change was -0.10 SDs/year (-1.01\%/year). White matter hyperintensity volume change was +0.11 SDs/year (+11.04\%/year). General FA change was -0.09 SDs/year. General MD change was $+0.34 \mathrm{SDs} / y e a r$. Each of the brain changes is illustrated in Fig. 3; although mean change was observed, there was substantial between-person variation in the magnitude of these changes.

The base models for general FA and MD had adequate fit to the data (general FA: $\chi^{2}(251)=639.44, p<0.001$, root mean square error of approximation $(\mathrm{RMSEA})=0.047$, Comparative Fit Index $(\mathrm{CFI})=0.914$, Tucker-Lewis Index $(\mathrm{TLI})=0.906$; general MD: $\chi^{2}(207)=618.86, p<0.001$, RMSEA $=0.059, \mathrm{CFI}=0.917, \mathrm{TLI}=0.907)$. Note that the base model for the three brain volumetric variables was saturated and thus had perfect fit to the data by definition. 
Table 1 Descriptive statistics and factor loadings for predictor variables

\begin{tabular}{|c|c|c|c|c|}
\hline Variable category & Measured variable & $n$ & Mean (SD) or $n$ for categorical measures & Factor loading \\
\hline \multirow[t]{3}{*}{ Physical fitness (latent) } & Grip strength & 823 & $28.54(9.39)$ & 0.393 \\
\hline & Forced expiratory volume & 856 & $2.30(0.68)$ & 0.458 \\
\hline & $6 \mathrm{~m}$ walk time & 860 & $4.35(1.32)$ & 0.482 \\
\hline \multirow[t]{8}{*}{ Allostatic load (latent) } & Fibrinogen & 819 & $3.25(0.61)$ & 0.482 \\
\hline & C-reactive protein & 830 & $4.94(7.84)$ & 0.506 \\
\hline & Interleukin-6 & 815 & $2.05(1.73)$ & 0.585 \\
\hline & Triglycerides & 832 & $1.65(0.82)$ & 0.314 \\
\hline & Glycated haemoglobin & 826 & $5.75(0.66)$ & 0.376 \\
\hline & Low-density lipoprotein & 829 & $2.93(1.04)$ & 0.251 \\
\hline & High-density lipoprotein & 832 & $1.46(0.44)$ & 0.341 \\
\hline & Body mass index & 866 & $27.92(4.45)$ & 0.402 \\
\hline \multirow[t]{3}{*}{ Socioeconomic status (latent) } & Father's occupational class $(1-5)^{*}$ & 960 & $2.91(0.94)$ & 0.372 \\
\hline & Own occupational class $(1-5)^{*}$ & 1091 & $3.54(1.20)$ & 0.505 \\
\hline & $\begin{array}{l}\text { Scottish Index of Multiple Deprivation } \\
(1-8)^{*}\end{array}$ & 1083 & $6.25(2.09)$ & 0.540 \\
\hline \multirow[t]{3}{*}{ Prior intelligence (latent) } & Moray House Test (age 11) & 1028 & $100(15.00)$ & 0.731 \\
\hline & NART (max. 50) & 864 & $34.38(8.18)$ & 0.958 \\
\hline & WTAR (max. 50) & 864 & $41.01(6.97)$ & 0.944 \\
\hline \multirow[t]{6}{*}{ Manifest (single) predictors } & Health conditions $(0-4)$ & 854 & $0.95(0.91)$ & - \\
\hline & Education (years)* & 1091 & $10.74(1.13)$ & - \\
\hline & Smoking & 866 & 415 never; 378 ex; 73 current & - \\
\hline & Alcohol (g per week)* & 928 & $11.98(16.79)$ & - \\
\hline & $A P O E \mathrm{e} 4$ & 1028 & $\begin{array}{l}306 \text { with } 1 \text { or } 2 \text { e } 4 \text { alleles; } 722 \text { with no e } 4 \\
\text { alleles }\end{array}$ & - \\
\hline & Polygenic risk for SCZ & 953 & $0.49(0.02)$ & - \\
\hline \multirow[t]{2}{*}{ Dementia screening } & MMSE age 73 (max. 30) & 865 & $28.75(1.42)$ & - \\
\hline & MMSE age 76 (max. 30) & 697 & $28.65(1.70)$ & - \\
\hline
\end{tabular}

*Variables collected at age 70; all other variables collected at age 73, unless otherwise noted

\section{Individual predictors of brain structure baseline level and change}

We regressed each brain baseline level and change variable on each of the predictors separately (also controlling for sex in each case; the effect of sex alone was also examined). The results of these analyses are shown in Fig. 4 (all values are provided in Supplementary Tables S1 for baseline level and Supplementary Table S2 for change). All results are reported with the brain outcomes scaled such that higher scores indicate healthier baseline levels (more grey and white matter, higher general FA, lower hyperintensity volume and lower general MD) and healthier changes (less decline in grey matter, white matter, and general FA; shallower increases in hyperintensity volume and general MD). That is, positive associations suggest healthier ageing and negative correlations suggest less healthy ageing.

Many associations were found between the predictors and brain baseline level in all five measures. The only variables that showed no significant relation with any baseline brain variable were alcohol consumption, $A P O E$ e4 status, and polygenic schizophrenia risk. We note that many of the predictor associations with brain baseline levels have been reported individually in previous publications on the Lothian Birth Cohort 1936 (Booth et al. 2015; Gow et al. 2012; Ritchie et al. 2015a, b); these results are provided here so as to evaluate multiple predictors together, and as a comparison to the new results for brain changes.

For change, there were far fewer associations. Only sex was associated with change in grey matter volume, with males declining faster. There were no significant relations with change in general FA. Some predictors had value, as shown in Fig. 4: physical fitness was significantly associated with healthier ageing in white matter volume and general MD, the $A P O E$ e4 allele was linked to less healthy changes in white matter and white matter hyperintensity volume, among some other, borderline associations. Importantly however, these results are uncorrected for multiple comparisons. Introducing a per-outcome false discovery rate correction (Benjamini and Hochberg 1995) reduced 
Table 2 Descriptive statistics and factor loadings for brain measurements

\begin{tabular}{|c|c|c|c|c|c|}
\hline \multirow[t]{2}{*}{ Measured variable } & \multicolumn{3}{|c|}{ Wave 2 (age 73) } & \multicolumn{2}{|c|}{ Wave 3 (age 76 ) } \\
\hline & $n$ & $\mathrm{M}(\mathrm{SD})$ & Factor loading & $n$ & $\mathrm{M}(\mathrm{SD})$ \\
\hline Grey matter volume $\left(\mathrm{cm}^{3}\right)$ & 657 & $472.43(44.68)$ & - & 461 & $465.67(43.61)$ \\
\hline White matter volume $\left(\mathrm{cm}^{3}\right)$ & 657 & $476.89(50.55)$ & - & 461 & $464.25(53.09)$ \\
\hline White matter hyperintensity volume $\left(\mathrm{cm}^{3}\right)$ & 656 & $12.23(12.18)$ & - & 464 & $15.85(14.57)$ \\
\hline Corpus callosum genu FA & 646 & $0.41(0.05)$ & 0.602 & 438 & $0.38(0.04)$ \\
\hline Corpus callosum splenium FA & 663 & $0.49(0.07)$ & 0.338 & 437 & $0.51(0.07)$ \\
\hline $\mathrm{L}$ arcuate fasciculus FA & 639 & $0.45(0.04)$ & 0.648 & 455 & $0.43(0.04)$ \\
\hline $\mathrm{R}$ arcuate fasciculus FA & 580 & $0.43(0.04)$ & 0.613 & 414 & $0.41(0.04)$ \\
\hline $\mathrm{L}$ anterior thalamic radiation $\mathrm{FA}$ & 556 & $0.32(0.03)$ & 0.642 & 429 & $0.33(0.03)$ \\
\hline $\mathrm{R}$ anterior thalamic radiation $\mathrm{FA}$ & 643 & $0.33(0.03)$ & 0.657 & 453 & $0.34(0.03)$ \\
\hline $\mathrm{L}$ rostral cingulum FA & 641 & $0.44(0.05)$ & 0.596 & 448 & $0.44(0.05)$ \\
\hline $\mathrm{R}$ rostral cingulum FA & 650 & $0.39(0.04)$ & 0.551 & 448 & $0.41(0.05)$ \\
\hline $\mathrm{L}$ uncinate fasciculus FA & 567 & $0.33(0.03)$ & 0.680 & 407 & $0.34(0.03)$ \\
\hline $\mathrm{R}$ uncinate fasciculus FA & 628 & $0.33(0.03)$ & 0.670 & 443 & $0.33(0.03)$ \\
\hline $\mathrm{L}$ inferior longitudinal fasciculus FA & 663 & $0.40(0.05)$ & 0.510 & 455 & $0.39(0.06)$ \\
\hline $\mathrm{R}$ inferior longitudinal fasciculus FA & 664 & $0.38(0.05)$ & 0.480 & 462 & $0.38(0.05)$ \\
\hline Corpus callosum genu MD & 646 & $770.05(66.05)$ & 0.659 & 438 & $850.28(82.99)$ \\
\hline Corpus callosum splenium MD & 663 & $978.47(173.32)$ & - & 437 & $852.01(153.16)$ \\
\hline $\mathrm{L}$ arcuate fasciculus MD & 639 & $659.50(47.60)$ & 0.686 & 455 & $699.45(58.14)$ \\
\hline $\mathrm{R}$ arcuate fasciculus MD & 580 & $646.35(52.29)$ & 0.715 & 414 & $681.46(58.21)$ \\
\hline $\mathrm{L}$ anterior thalamic radiation $\mathrm{MD}$ & 556 & $758.24(66.00)$ & 0.653 & 429 & $795.90(66.85)$ \\
\hline $\mathrm{R}$ anterior thalamic radiation $\mathrm{MD}$ & 643 & $754.29(62.44)$ & 0.738 & 453 & $791.45(86.75)$ \\
\hline $\mathrm{L}$ rostral cingulum MD & 641 & $648.13(45.64)$ & 0.557 & 448 & $673.84(46.71)$ \\
\hline $\mathrm{R}$ rostral cingulum MD & 650 & $651.92(45.08)$ & 0.618 & 448 & $663.39(42.12)$ \\
\hline $\mathrm{L}$ uncinate fasciculus MD & 567 & $770.37(53.82)$ & 0.643 & 407 & $794.30(57.22)$ \\
\hline $\mathrm{R}$ uncinate fasciculus MD & 628 & $756.31(52.30)$ & 0.703 & 443 & $796.54(58.11)$ \\
\hline L inferior longitudinal fasciculus MD & 663 & $771.85(100.10)$ & 0.426 & 455 & $826.54(143.47)$ \\
\hline $\mathrm{R}$ inferior longitudinal fasciculus MD & 664 & $772.20(101.48)$ & 0.414 & 462 & $801.15(122.90)$ \\
\hline
\end{tabular}

Factor loadings were invariant across waves. The splenium had a low loading for MD and so was not included in the general factor

$F A$ fractional anisotropy; $M D$ mean diffusivity; $L / R$ left/right hemisphere

Table 3 Associations (standardized betas) among latent and manifest predictor variables, estimated within a structural equation model

\begin{tabular}{|c|c|c|c|c|c|c|c|c|c|c|}
\hline Variable & 1. & 2. & 3. & 4. & 5. & 6. & 7. & 8. & 9. & 10. \\
\hline 1. Sex (male) & - & & & & & & & & & \\
\hline 2. Physical fitness ${ }^{\dagger}$ & 0.01 & - & & & & & & & & \\
\hline 3. Allostatic load ${ }^{\dagger}$ & -0.02 & $-0.68^{* * *}$ & - & & & & & & & \\
\hline 4. Health conditions & $0.11^{* *}$ & $-0.36^{* * *}$ & $0.37^{* * * *}$ & - & & & & & & \\
\hline 5. Socioeconomic status ${ }^{\dagger}$ & 0.05 & $0.38^{* * *}$ & $-0.35^{* * *}$ & $-0.11^{*}$ & - & & & & & \\
\hline 6. Prior intelligence ${ }^{\dagger}$ & $-0.09^{* *}$ & $0.26^{* * *}$ & $-0.20^{* * *}$ & $-0.09^{* *}$ & $0.69^{* * *}$ & - & & & & \\
\hline 7. Education & 0.01 & $0.16^{* *}$ & $-0.17^{* * *}$ & $-0.08^{*}$ & $0.71^{* * * *}$ & $0.55^{* * *}$ & - & & & \\
\hline 8. Smoking & $-0.08^{*}$ & $-0.27^{* * *}$ & $0.25^{* * *}$ & 0.05 & $-0.21^{* * *}$ & $-0.07^{*}$ & $-0.11^{* *}$ & - & & \\
\hline 9. Alcohol consumption & $0.35^{* * *}$ & $0.12^{*}$ & $-0.18^{* * *}$ & -0.05 & $0.35^{* * * *}$ & $0.19^{* * * *}$ & $0.19^{* * *}$ & 0.03 & - & \\
\hline 10. $A P O E$ e 4 & 0.04 & 0.08 & -0.07 & -0.03 & 0.04 & 0.03 & 0.002 & -0.06 & 0.03 & - \\
\hline 11. Polygenic risk for schizophrenia & 0.04 & -0.03 & -0.02 & -0.04 & -0.003 & -0.07 & -0.02 & 0.06 & 0.06 & -0.01 \\
\hline
\end{tabular}

$* p<0.05, * *=p<0.01 ; * * *=p<0.001{ }^{\dagger}=$ latent variable predictor 
Fig. 3 Change in each brain measure between age 73 and age 76 years, showing an individual point for each participant at the first scanning wave (left, red cluster) and the second (right, purple cluster). Participants who returned for the second scan have their points connected with a grey line. For comparison, all measurements are shown on a standardized scale

all change associations to non-significance except the association of sex with grey matter change, and the association of physical fitness with change in general MD (see dagger signs in Fig. 4).

We next explored the degree of correspondence between predictors of level and predictors of change. We did this in two ways. First, for those predictors that were significant (uncorrected) for either level or change, we tested whether the effect sizes for level and for change were significantly different from one another. As shown by the 'a' superscript in Fig. 4, we found that many of the associations that were significant for level were significantly different from the corresponding association for change (for example, the associations of SES, prior intelligence, and education with the volumetric measures). Some of the significant associations with change (namely the APOE e4 allele and the schizophrenia polygenic profile score) were significantly larger than those for level. Second, we tested the correlation of the vector of effect sizes for baseline level with the vector of effect sizes for change. Across all effect sizes for all brain variables, there was only a modest, non-significant correlation between the effect sizes $(r(53)=0.27, p=0.05)$, suggesting relatively poor correspondence between the effect sizes. Overall, this indicated that obtaining a significant effect for a correlate of levels of late life brain structure cannot be regarded as prima facie evidence that the variable is a similarly-effective predictor of ageing-related structural change.

\section{Models with multiple predictors of baseline brain structure and change}

The elastic net regression procedure selected variables to include in the simultaneous-predictor models, keeping each model as parsimonious as possible (Table 4). As shown previously (Morozova et al. 2015), penalized regression procedures often select variables that are not incrementally significant in a regression model, but that nonetheless reduce the mean squared error if they are included. This was the case for our models: the selection procedure selected several variables that were not significant in the model (the unbolded coefficients in Table 4). With only a few differences, the change results from the individual analyses remained intact in these simultaneous models: physical fitness and $A P O E$ e4 status remained the most substantial predictors of brain
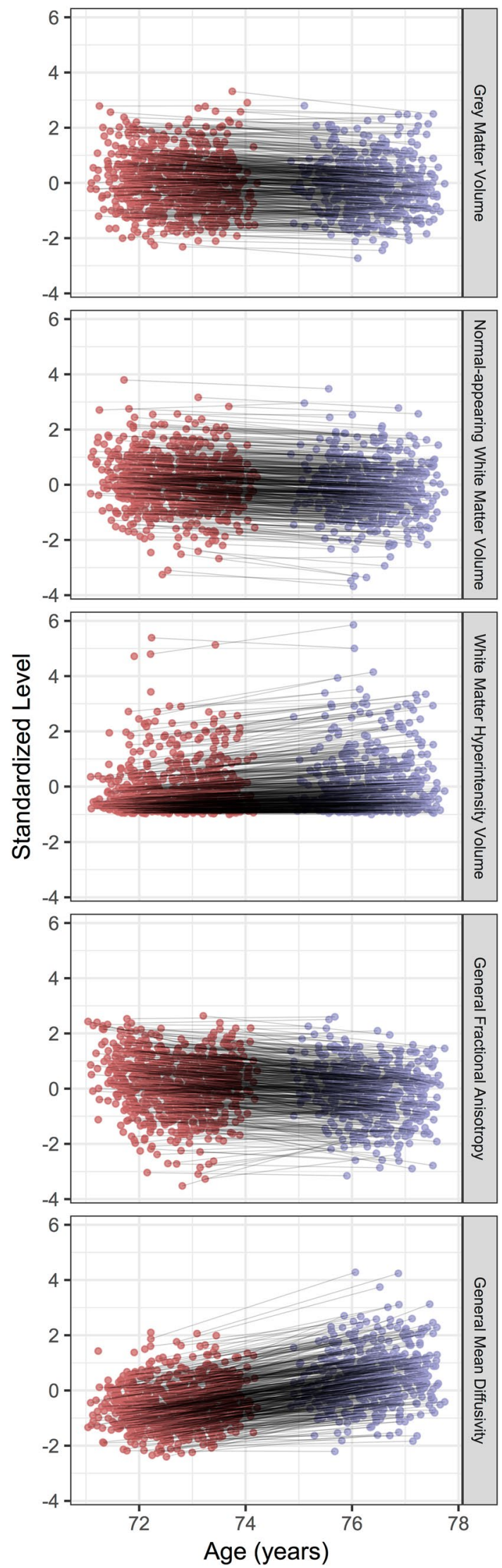


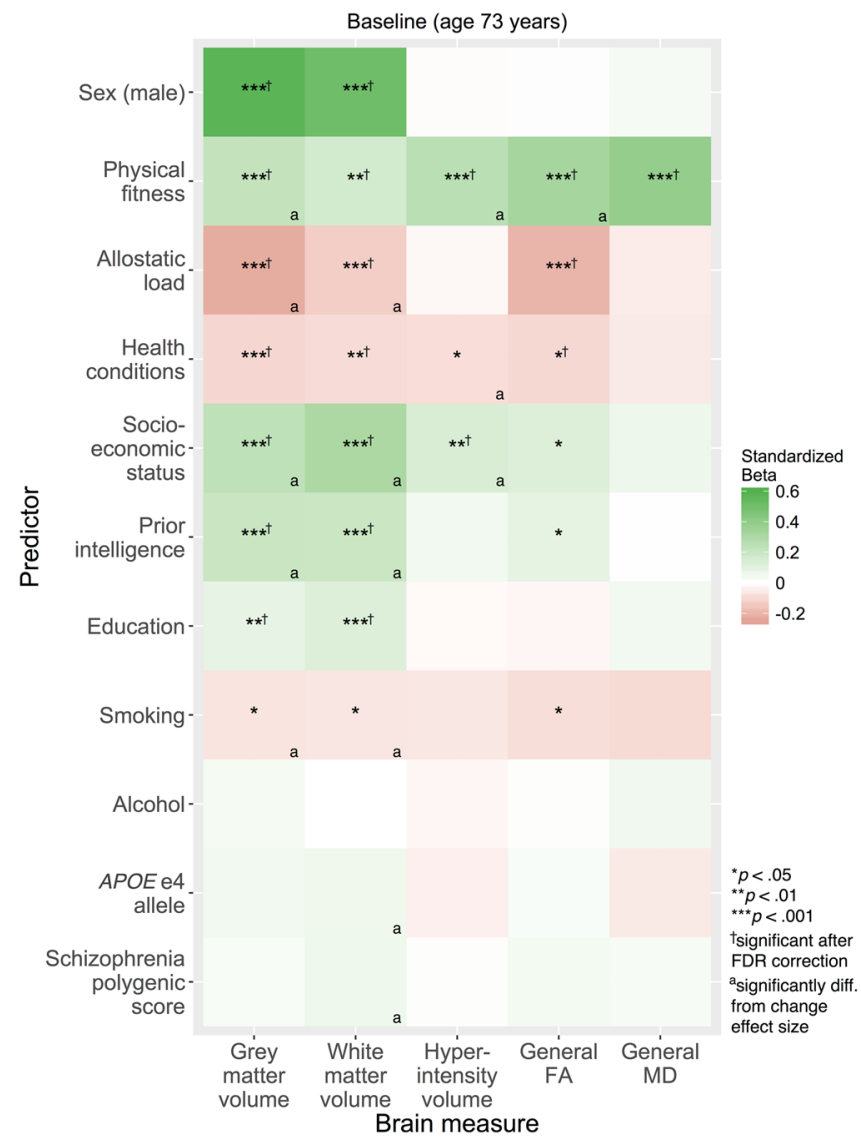

Fig. 4 Associations of each predictor with baseline levels of (left) and changes in (right) each brain measure (all associations adjusted for age and sex). Brain variables scaled such that positive associations (green) indicate healthier brain baseline levels and brain ageing, and negative associations (red) indicate unhealthier brain baseline lev-

change, although some factors that were not previously statistically significant, such as alcohol consumption (in this case associated with white matter hyperintensity progression) became significant in the simultaneous model.

\section{Models excluding individuals with possible dementia}

Finally, we re-ran our individual-predictor-variable analyses removing sixteen individuals with mini-mental state examination (Folstein et al. 1975) scores below 24 (a commonly-used cut-off that might indicate mild cognitive impairment or dementia) at either testing wave. Whereas the vast majority of the results were very similar to those from the main analysis, there were no longer any significant baseline level associations for smoking, or any significant change associations for possession of the $A P O E$ e 4 allele.

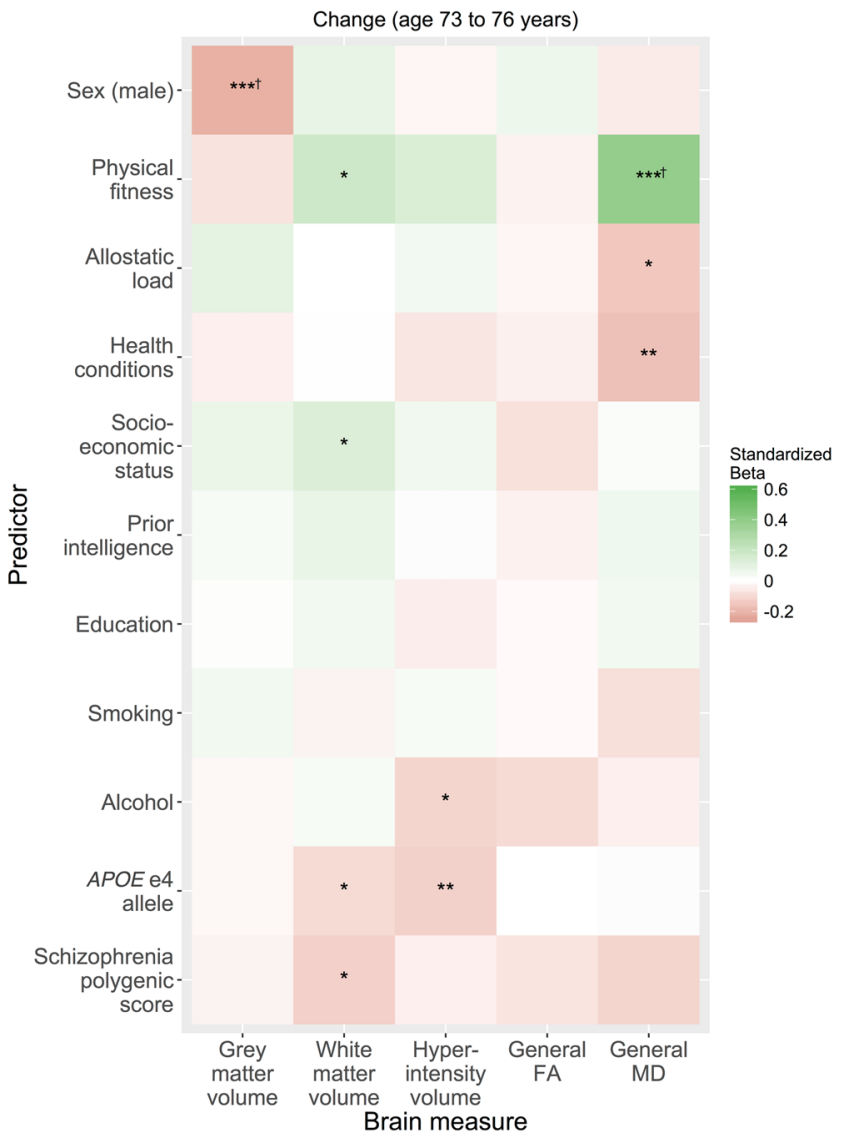

els and brain ageing. Hyperintensity = white matter hyperintensity; $F A$ fractional anisotropy; $M D$ mean diffusivity. ${ }^{*} p<0.05,{ }^{* *} p<0.01$, ${ }^{* * * *} p<0.001$; ${ }^{\dagger}$ significant after false discovery rate (FDR) correction; a effect size for baseline was significantly different from effect size for change

\section{Discussion}

This investigation of neurostructural changes between age 73 and age 76 makes two key contributions. First, it identifies some potentially important predictors of brain change. For example, physical fitness and $A P O E$ e 4 were the most consistent predictors of differential rates of brain ageing, and are therefore promising foci for further investigation into predictions of brain change. On the whole, effect sizes for predictors of longitudinal brain change were small (all standardized $\beta$ values $<0.4$ for brain changes), and few were statistically significant. Predictors differed across the different neural outcomes under study, suggesting that ageing-related brain change is a multifaceted, multidetermined process (see e.g. Kievit et al. 2014). Second, the results highlight the important point that variables that are significantly associated crosssectionally with baseline levels of neural structure do not necessarily make significant predictions about longitudinal neural changes. We found that many of the correlates 


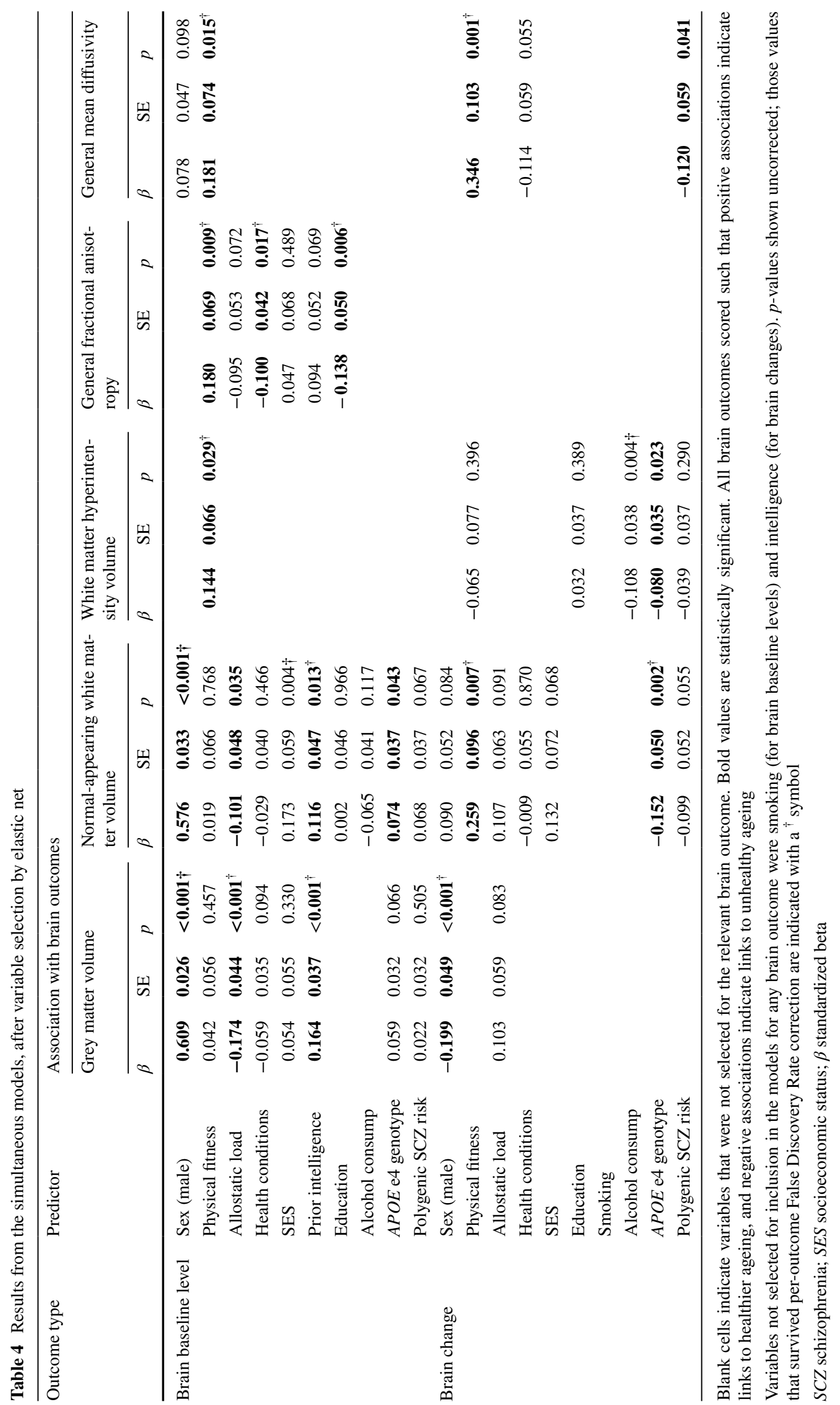


of initial baseline levels of brain structure did not significantly predict subsequent rates of change in brain structure; in some cases predictor associations with change were in opposite directions from associations with baseline levels.

The fact that significant predictors of longitudinal brain changes were not always correlates of baseline levels of brain structure suggests that variables we found to be related to brain structure longitudinally but not crosssectionally (such as $A P O E$ e 4 and polygenic schizophrenia risk; though note that these were both weak associations that did not survive multiple comparisons correction) are, if causal, only likely to operate in old age, since they had not had any appreciable associations with brain structure preceding the follow-up waves of our study. Variables such as education and prior intelligence, which were related crosssectionally but not longitudinally to brain structure, might represent correlates of developmental changes in brain structure from earlier in life, but appear not to be prospectively coupled with ageing-related changes in the indices of brain structure examined here.

Physical fitness was significantly related to healthier brain ageing in three of the five measures included here, and two of these relations (to changes in normal-appearing white matter and in general mean diffusivity) survived both the multivariate model and the MMSE exclusion. It was also one of the few variables that had a significant correlation both with baseline levels and with changes in multiple brain measures. Such results are in line with the literature on the effects of physical fitness on brain (and cognitive) ageing, some of which comes from randomized experiments and can thus test causality (Ahlskog et al. 2011). One caveat should be noted: whereas the measures we used (grip strength, lung function, and walk speed) are excellent general indicators of physical fitness (Enright et al. 2003), they may - at least partially — reflect pre-existing differences in bodily integrity (Frederiksen et al. 2002). The results do not, therefore, mean that improving physical fitness in old age will necessarily improve brain health, although they do leave open that possibility. Nevertheless, whether or not the relations we found are causal, our results show that differences in physical fitness may be informative for the prediction of future brain ageing.

Sex differences were found for grey matter both at baseline and in terms of change, but they ran in the opposite direction: males had significantly larger grey matter volume at baseline (as expected on the basis of prior studies: see the meta-analysis by Ruigrok et al. 2014), but also showed significantly greater loss of grey matter across the subsequent 3 years than females. This may be due to the 'law of initial value' (that the degree of change is affected by the baseline level; in this case, that individuals with initiallyhigher levels of a trait have 'more to lose' subsequently), or could represent a sex-specific ageing process, affecting males disproportionately during this period of life.

The two genetic factors we studied, the APOE e4 allele and polygenic risk for schizophrenia, showed small-sized relations to neurostructural decline. $A P O E$ e4 was linked to significantly faster decline in white matter volume and significantly faster accumulation of white matter hyperintensities (though this association did not survive multiple comparisons correction). As noted above, there has previously been mixed evidence for any detectable influence of $A P O E$ e 4 on brain decline in healthy individuals (Josephs et al. 2008; Wishart et al. 2006), despite its being a wellreplicated risk factor for Alzheimer's-related brain pathology (Liu et al. 2015). It should be noted that the effects of $A P O E$ e 4 were smaller in the analysis excluding individuals with low MMSE scores, implying that any $A P O E$ effects are stronger in individuals whose MMSE scores might indicate prodromal dementia (though the significant effects were small in the full sample, so this may also reflect the lower power of the secondary analysis). To our knowledge, no studies have tested the association of polygenic schizophrenia risk with longitudinal brain change. Suggestive evidence from the present study hints that polygenic schizophrenia risk may be a useful predictor of subsequent decline in brain structure (the relation did not survive multiple comparisons correction); if replicated, the reasons for this-a shared genetic aetiology (Lopez et al. 2015), or influences of schizophrenia genes on lifestyle factors that are detrimental to brain maintenance-would be uncertain. The polygenic schizophrenia risk score was chosen on the basis of work showing genetic correlations between schizophrenia and cognitive abilities, including in older age (Hagenaars et al. 2016; there have also been previous cognitive results in this sample; McIntosh et al. 2013), but polygenic risk scores for other conditions, such as stroke, may also be predictive of neurostructural changes and should be a target for future work.

Rather than relying on conventional multiple regression methods, which have biases with respect to overfitting (particularly in the presence of high multicollinearity), we used penalized regression-designed to reduce overfit-in our simultaneous models. Future studies of ageing-related brain changes could use our multivariate findings as a basis for investigations to more directly determine the replicability of these results.

All of the structural brain parameters we measured have previously been related both to baseline levels of and changes in at least some key cognitive functions, such as memory, reasoning, and processing speed (e.g. Charlton et al. 2010; Lövdén et al. 2014; Ritchie et al. 2015a, b). Future work should use more complex structural equation models to simultaneously investigate predictors of brain change alongside predictors of cognitive change: 
for instance, researchers could test whether fitness-related white matter changes mediate simultaneous changes in cognitive ability. Such an analysis, which is beyond the scope of the present paper, would take a step closer to a causal understanding of the antecedents and brain mechanisms of ageing-related cognitive decline (see Köhncke et al. 2016 for an example of this design with engagement in leisure activities as the predictor).

The present study is, to our knowledge, the largest-scale investigation of predictors of differential brain ageing to date. Compared to previous longitudinal studies of this nature (e.g. Enzinger et al. 2005), our sample was particularly large, enough to reduce (though not eliminate) the impact of scanner measurement noise on our estimates. We also assessed a large number of of predictors and structural indices, adding to our study's comprehensiveness. Moreover, the narrow age range meant that the confounding effects of age were substantially reduced, and the cultural and ethnic homogeneity of the cohort limited other confounding effects. Where appropriate, we capitalized on latent variable modeling, which reduced the dimensionality of our predictors and eliminated measurement error (for instance, the use of general factors of FA and MD removed the influence of tract-specific measurement error), implementing latent difference score models of change over time.

The study has some limitations. First, our study period only lasted 3 years: such a brief period may not have resulted in substantial enough changes for us to reliably detect predictors with smaller effect sizes, even with our relatively large sample size (it is likely that the majority of the effects of these predictors are small); two waves of measurement also prevented us from examining any nonlinearities in brain change. Second, healthier individuals are more likely to enroll in studies of this nature, and are less likely to drop out as the study continues. We implemented maximum likelihood estimation methods that reduce missing data bias resulting from longitudinal attrition, under the assumption that patterns of missingness in our outcomes that systematically relate to the unobserved scores on those outcomes are accounted for by non-missing scores on the variables included in our model. Nevertheless, restriction of range in baseline levels of our variables meant that we likely underestimated many effect sizes reported here. We might, for instance, have missed particularly debilitating brain change effects in individuals with very high baseline levels of allostatic load.

Third, the brain variables we used were relatively broad-brush: they indexed change in global grey and white matter, and in general white matter microstructure, but further research with finer-grained brain measures-examining particular grey matter regions or specific white matter tracts, which may themselves be more strongly linked to certain cognitive abilities-is required to test hypotheses relating potential predictors to specific brain areas (Maass et al. 2015). Fourth, our predictors were all time-invariant: we focused on the predictive value of measurements made at age 73 for declines in neural health between 73 and 76 . However, a number of the parameters-such as physical fitness, allostatic load, and alcohol consumption-also change over time. Future studies should investigate whether changes in these parameters are coupled with changes in any of the brain markers assessed here. Additional waves of follow-up in the LBC1936 should allow us greater power to detect correlations with time-varying covariates (Rast and Hofer 2014).

This study investigated predictors of differential brain ageing between age 73 and 76 years. There was little correspondence between variables that were cross-sectionally associated with brain variables and those that predicted their changes, though physical fitness-among the strongest predictors in our analyses-was an exception. These results serve as a benchmark for future studies that attempt to replicate and validate the contributions of predictors of brain maintenance (Nyberg et al. 2012), and to estimate the extent to which their proximal effects on different brain structures mediate their ultimate effects on cognitive and functional decline.

Acknowledgements We thank the LBC1936 members for their participation. We thank Catherine Murray, Caroline Brett, and Ross Henderson for data collection and data entry; the nurses and other staff at the Wellcome Trust Clinical Research Facility and the radiographers at the Brain Research Imaging Centre for acquisition of all MRI data; and the staff at Lothian Health Board and at the Scottish Council for Research in Education (SCRE) Centre, University of Glasgow. LBC1936 data collection was supported by the Disconnected Mind project, funded by Age UK. J.M.W. is part-funded by the Scottish Funding Council as part of the SINAPSE Collaboration (http://www. sinapse.ac.uk). The work was undertaken by The University of Edinburgh Centre for Cognitive Ageing and Cognitive Epidemiology, part of the cross council Lifelong Health and Wellbeing Initiative (MR/ K026992/1). Funding from the Biotechnology and Biological Sciences Research Council (BBSRC) and Medical Research Council (MRC) is gratefully acknowledged. Some of the work on this paper was carried out with the assisance of a Boehringer Ingelheim Fonds travel grant to author S.J.R. None of the authors have relevant conflicts of interest to declare.

Open Access This article is distributed under the terms of the Creative Commons Attribution 4.0 International License (http:// creativecommons.org/licenses/by/4.0/), which permits unrestricted use, distribution, and reproduction in any medium, provided you give appropriate credit to the original author(s) and the source, provide a link to the Creative Commons license, and indicate if changes were made.

\section{References}

Ahlskog JE, Geda YE, Graff-Radford NR, Petersen RC (2011) Physical exercise as a preventive or disease-modifying treatment of dementia and brain aging. Mayo Clin Proc 86:876-884 
Behrens TEJ, Berg HJ, Jbabdi S, Rushworth MF, Woolrich MW (2007) Probabilistic diffusion tractography with multiple fibre orientations. What can we gain?. NeuroImage 23:144-155

Bender AR, Prindle JJ, Brandmaier AM, Raz N (2016) White matter and memory in healthy adults: coupled changes over 2 years. NeuroImage. doi:10.1016/j.neuroimage.2015.10.085

Benjamini Y, Hochberg Y (1995) Controlling the false discovery rate: a practical and powerful approach to multiple testing. J Royal Stat Soc B 57:289-300

Booth T, Royle NA, Corley J, Gow AJ, Valdés Hernández MC, Muñoz Maniega S, Ritchie SJ, Bastin ME, Starr JM, Wardlaw JM, Deary IJ (2015) Association of allostatic load with brain structure and cognitive ability in later life. Neurobiol Aging 36:1390-1399

Brayne C (2007) The elephant in the room-healthy brains in later life, epidemiology and public health. Nat Rev Neurosci 8:233-239

Charlton RA, Schiavone F, Barrick TR, Morris RG, Markus HS (2010) Diffusion tensor imaging detects age related white matter change over a 2 year follow-up which is associated with working memory decline. J Neurol Psychiatr 81:1319

Clayden J, Storkey A, Muñoz Maniega S, Bastin ME (2009) Reproducibility of tract segmentation between sessions using an unsupervised modelling-based approach. NeuroImage 45:377-385

Clayden JD, Muñoz Maniega S, Storkey AJ, Martin MD, Bastin ME, Clark CA (2011) TractoR: magnetic resonance imaging and tractography with R. J Stat Soft 44:1-18

Deary IJ, Gow AJ, Taylor MD, Corley J, Brett C, Wilson V, Campbell H, Whalley LJ, Visscher PM, Porteous DJ, Starr JM (2007) The Lothian Birth Cohort 1936: a study to examine influences on cognitive ageing from age 11 to age 70 and beyond. BMC Geriatr 7:28

Deary IJ, Gow AJ, Pattie A, Starr JM (2012) Cohort profile: the Lothian Birth Cohorts of 1921 and 1936. Int J Epidemiol 41:1576-1584

Enright PL, McBurnie MA, Bittner V, Tracy RP, McNamara R, Arnold A, Newman AB (2003) The 6-min walk test: a quick measure of functional status in elderly adults. CHEST J 123:387-398

Enzinger C, Fazekas F, Matthews PM, Ropele S, Schmidt H, Smith S, Schmidt R (2005) Risk factors for progression of brain atrophy in aging: 6-year follow-up of normal subjects. Neurology 64:1704-1711

Folstein MF, Folstein SE, McHugh PR (1975) "Mini-mental state": a practical method for grading the cognitive state of patients for the clinician. J Psychiatr Res 12:189-198

Fotenos AF, Snyder AZ, Girton LE, Morris JC, Buckner RL (2005) Normative estimates of cross-sectional and longitudinal brain volume decline in aging and AD. Neurology 64:1032-1039

Frederiksen H, Galst D, Petersen HC, Hjelmborg J, McGue M, Vaupel JW, Christensen K (2002) Hand grip strength: a phenotype suitable for identifying genetic variants affecting mid-and latelife physical functioning. Genet Epidemiol 23:110-122

Friedman J, Hastie T, Tibshirani R (2010) Regularization paths for generalized linear models via coordinate descent. J Stat Soft 33:1-22

Gow AJ, Bastin ME, Muñoz Maniega S, Valdés Hernández S, Morris Z, Murray C, Royle NA, Starr JM, Deary IJ, Wardlaw JM (2012) Neuroprotective lifestyles and the aging brain activity, atrophy, and white matter integrity. Neurology 79:1802-1808

Hagenaars SP, Harris SE, Davies G, Hill WD, Liewald DC, Ritchie SJ, Deary IJ (2016) Shared genetic aetiology between cognitive functions and physical and mental health in UK Biobank $(N=$ 112151 ) and 24 GWAS consortia. Mol Psychiatr 21:1624-1632

Hsu J-L, van Hecke W, Bai C-H, Lee C-H, Tsai Y-H, Chiu H-C, Jaw F-S, Hsu C-Y, Leu J-G, Chen W-H, Leemans A (2010)
Microstructural white matter changes in normal aging: a diffusion tensor imaging study with higher-order polynomial regression models. NeuroImage 49:32-43

Hudkins M, O’Neill J, Tobias MC, Bartzokis G, London ED (2012) Cigarette smoking and white matter microstructure. Psychopharmacol 221:285-295

Jenkinson M, Beckmann CF, Behrens TJ, Woolrich MW, Smith SM (2012) FSL. NeuroImage 62:782-90

Josephs KA, Whitwell JL, Ahmed Z, Shiung MM, Weigand SD, Knopman DS, Boeve BF, Parisi JE, Petersen RC, Dickson DW, Jack CR Jr (2008) $\beta$-amyloid burden is not associated with rates of brain atrophy. Ann Neurol 63:204-212

Kievit RA, Davis SW, Mitchell DJ, Taylor JR, Duncan J, Cam-CAN Research team, Henson RN (2014) Distinct aspects of frontal lobe structure mediate age-related differences in fluid intelligence and multitasking. Nat Commun 5:5658

Kloppenborg RP, Nederkoorn PJ, Geerlings MI, van den Berg E (2014) Presence and progression of white matter hyperintensities and cognition: a meta-analysis. Neurology 82:2127-2138

Kochunov P, Williamson DE, Lancaster J, Fox P, Cornell J, Blangero J, Glahn DC (2012) Fractional anisotropy of water diffusion in cerebral white matter across the lifespan. Neurobiol Aging 33:9-20

Köhncke Y, Laukka EJ, Brehmer Y, Kalpouzos G, Li TQ, Fratiglioni L, Lövdén M (2016) Three-year changes in leisure activities are associated with concurrent changes in white matter microstructure and perceptual speed in individuals aged 80 years and older. Neurobiol Aging 41:173-186

Laukka EJ, Lövdén M, Kalpouzos G, Papenberg G, Keller L, Graff C, Li T-Q, Fratiglioni L, Bäckman L (2015) Microstructural white matter properties mediate the association between APOE and perceptual speed in very old persons without dementia. Plos One 10:e0134766

Liu Y, Yu JT, Wang HF, Han PR, Tan CC, Wang C, Meng XF, Riascher SL, Saykin AJ, Tan L (2015) APOE genotype and neuroimaging markers of Alzheimer's disease: systematic review and meta-analysis. J Neurol Neurosurg Psychiatr 86:127-134

Liu B, Zhang X, Cui Y, Qin W, Tao Y, Li J, Jiang T (2016) Polygenic risk for schizophrenia influences cortical gyrification in 2 independent general populations. Schiz Bull doi:10.1093/schbul/ sbw051

Lopez LM et al (2015) Genes from a translational analysis support a multifactorial nature of white matter hyperintensities. Stroke 46:341-347

Lövdén M, Köhnke Y, Laukka EJ, Kalpouzous G, Salami A, Li T-Q, Fratiglioni L, Bäckman L (2014) Changes in perceptual speed and white matter microstructure in the corticospinal tract are associated in very old age. NeuroImage 102:520-530

Maass A, Düzel S, Brigadski T, Goerke M, Becke A, Sobieray U, Neumann K, Lövdén M, Lindenberger U, Bäckman L, BraunDullaeus R, Ahrens D, Heinze HJ, Müller NG, Lessmann V, Sendtner M, Düzel E (2015) Relationships of peripheral IGF-1, VEGF and BDNF levels to exercise-related changes in memory, hippocampal perfusion and volumes in older adults. NeuroImage. doi:10.1016/j.neuroimage.2015.10.084

McArdle JJ (2009) Latent variable modeling of differences and changes with longitudinal data. Ann Rev Psychol 60:577-605

McEwen BS (1998) Stress, adaptation, and disease: allostasis and allostatic load. Ann NY Acad Sci 840:33-44

McIntosh AM, Gow AJ, Luciano M, Davies G, Liewald DC, Harris SE, Corley J, Hall J, Starr JM, Porteus DJ, Tenesa A, Visscher PM, Deary IJ (2013) Polygenic risk for schizophrenia is associated with cognitive change between childhood and old age. Biol Psychiatr 73:938-943

Morozova O, Levina O, Uusküla A, Heimer R (2015) Comparison of subset selection methods in linear regression in the context 
of health-related quality of life and substance abuse in Russia. BMC Med Res Methodol 15:71

Muñoz Maniega S, Chappell FM, Valdés Hernández MC, Armitage PA, Makin SD, Heye AK, Thrippleton MJ, Sakka E, Shuler K, Dennis MS, Wardlaw JM (In Press) Integrity of normal-appearing white matter: influence of age, visible lesion burden and hypertension in patients with small vessel disease. J Cereb Blood Flow Metab. doi:10.1177/0271678X16635657

Muthén LK, Muthén BO (1998-2014) Mplus User's Guide: The Comprehensive Modeling Program for Applied Researchers. (Muthén and Muthén)

Nelson HE, Willison JR (1991) National Adult Reading Test (NART). Windsor: Nfer-Nelson

Nyberg L, Lövdén M, Riklund K, Lindenberger U, Bäckman L. (2012) Memory aging and brain maintenance. Trend Cognitive Sci 16:292-305

Oscar-Berman M, Marinković K (2007) Alcohol: effects on neurobehavioral functions and the brain. Neuropsychol Rev 17:239-257

Qiu C, Sigurdsson A, Zhang Q, Jonsdottir MK, Kjartansson O, Eiriksdottir G, Garcia ME, Harris TB, van Buchem MA, Gudnason V, Launer LJ (2014) Diabetes, markers of brain pathology and cognitive function. Ann Neurol 75:138-146

Rast P, Hofer SM (2014) Longitudinal design considerations to optimize power to detect variances and covariances among rates of change: simulation results based on actual longitudinal studies. Psychol Methods 19:133-154

Raz N, Rodrigue KM, Acker JD (2003) Hypertension and the brain: vulnerability of the prefrontal regions and executive functions. Behav Neurosci 117:1169-1180

Ritchie SJ, Bastin ME, Tucker-Drob EM, Muñoz Maniega S, Engelhardt LE, Cox SR, Royle NA, Gow AJ, Corley J, Pattie A, Taylor AM, Valdés Hernández MC, Starr JM, Wardlaw JM, Deary IJ (2015a) Coupled changes in brain white matter microstructure and fluid intelligence in later life. J Neurosci 35:8672-8682

Ritchie SJ, Dickie DA, Cox SR, Valdés Hernández MC, Corley J, Royle NA, Pattie A, Aribisala BS, Redmond P, Muñoz Maniega S, Taylor AM, Sibbett R, Gow AJ, Starr JM, Bastin ME, Wardlaw JM, Deary IJ (2015b) Brain volumetric changes and cognitive ageing during the eighth decade of life. Hum Brain Mapp 36:4910-4925

Ruigrok AN, Salimi-Khorshidi G, Lai MC, Baron-Cohen S, Lombardo MV, Tait RJ, Suckling J (2014) A meta-analysis of sex differences in human brain structure. Neurosci Biobehav Rev 39:34-50

Salthouse TA (2011) Neuroanatomical substrates of age-related cognitive decline. Psychol Bull 137:753-784

Schizophrenia Working Group of the Psychiatric Genomics Consortium (2014) Biological insights from 108 schizophrenia-associated genetic loci. Nature 511:421-427

Schmidt R, Ropele S, Enzinger C, Petrovic K, Smith S, Schmidt H, Matthews PM, Fazekas F (2005) White matter lesion progression, brain atrophy, and cognitive decline: the Austrian stroke prevention study. Ann Neurol 58:610-616

Scottish Council for Research in Education (1949) The trend of scottish intelligence: a comparison of the 1947 and 1932 surveys of the intelligence of 11-year-old pupils (University of London Press)
Scottish Executive (2006) Scottish Index of Multiple Deprivation 2006 (Scottish Executive)

Sexton CE, Betts JF, Demnitz N, Dawes H, Ebmeier KP, JohansenBerg H (2015) A systematic review of MRI studies examining the relationship between physical fitness and activity and the white matter of the ageing brain. NeuroImage. doi:10.1016/j. neuroimage.2015.09.071

Staal WG, Hulshoff Pol HE, Schnack HG, Hoogendoorn MLC, Jellema K, Kahn RS (2000) Structural brain abnormalities in patients with schizophrenia and their healthy siblings. Am J Psychiatr 157:416-421

Tian Q, Studenski SA, Resnick SM, Davatzikos C, Ferrucci L (2015) Midlife and late-life cardiorespiratory fitness and brain volume changes in late adulthood: results from the Baltimore Longitudinal Study of Aging. J Gerontol A Biol Sci Med Sci. doi:10.1093/ erona/glv041

Tucker-Drob EM, Salthouse TA (2011) Individual differences in cognitive aging. In: Chamorro-Premuzic T, von Stumm S, Furnham A (eds). The wiley-blackwell handbook of individual differences, 1st edn. (Wiley-Blackwell), p 242-267

Valdés Hernández MC, Ferguson KJ, Chappell FM, Wardlaw JM (2010) New multispectral MRI data fusion technique for white matter lesion segmentation: method and comparison with thresholding in FLAIR images. Eur Radiol 20:1684-1691

Van der Auwera S, Wittfeld K, Homuth G, Teumer A, Hegenscheid K, Grabe HJ (2015) No association between polygenic risk for schizophrenia and brain volume in the general population. Biol Psychiatr. doi:10.1016/j.biopsych.2015.02.038

Wang X, Valdés Hernández MC, Doubal F, Chappell FM, Wardlaw JM (2012) How much do focal infarcts distort white matter lesions and global cerebral atrophy measures? Cerebrovasc Dis 34:336-342

Wardlaw JM, Bastin ME, Valdés Hernándes MC, Muñoz Maniega S, Royle NA, Morris Z, Clayden JD, Sanderman EM, Eadie E, Murray C, Starr JM, Deary IJ (2011) Brain aging, cognition in youth and old age and vascular disease in the Lothian Birth Cohort 1936: rationale, design and methodology of the imaging protocol. Int J Stroke 6:547-559

Wardlaw JM et al (2013) Neuroimaging standards for research into small vessel disease and its contribution to ageing and neurodegeneration. Lancet Neurol 12:822-838

Wardlaw JM, Valdés Hernández MC, Muñoz Maniega S (2015) What are white matter hyperintensities made of? Relevance to vascular cognitive impairment. J Am Heart Assoc 4:e01140

Wechsler D (2001) Wechsler test of adult reading: WTAR (psychological corporation)

Widaman KF, Ferrer E, Conger RD (2010) Factorial invariance within longitudinal structural equation models: Measuring the same construct across time. Child Dev Perspect 4:10-18

Wishart HA, Saykin AJ, McAllister TW, Rabin LA, McDonald BC, Flashman LA, Roth RM, Mamourian AC, Tsongalis GJ, Rhodes CH (2006) Regional brain atrophy in cognitively intact adults with a single APOE $\varepsilon 4$ allele. Neurology 67:1221-1224

Zou H, Hastie T (2005) Regularization and variable selection via the elastic net. J Royal Stat Soc B 67:301-320 